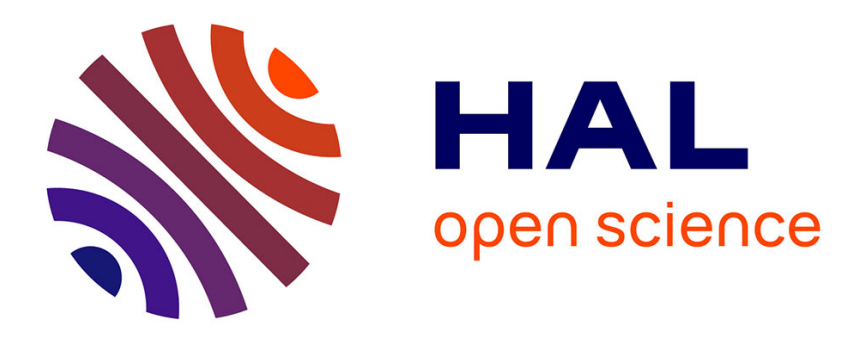

\title{
Excitonic Mechanism of Local Phase Transformations by Optical Pumping
}

\author{
Serguei Brazovskii, Natasha Kirova
}

\section{To cite this version:}

Serguei Brazovskii, Natasha Kirova. Excitonic Mechanism of Local Phase Transformations by Optical Pumping. Journal of Superconductivity and Novel Magnetism, 2014, 27, pp.1009 - 1013. 10.1007/s10948-013-2432-9 . hal-01108706

\section{HAL Id: hal-01108706 \\ https://hal.science/hal-01108706}

Submitted on 23 Jan 2015

HAL is a multi-disciplinary open access archive for the deposit and dissemination of scientific research documents, whether they are published or not. The documents may come from teaching and research institutions in France or abroad, or from public or private research centers.
L'archive ouverte pluridisciplinaire HAL, est destinée au dépôt et à la diffusion de documents scientifiques de niveau recherche, publiés ou non, émanant des établissements d'enseignement et de recherche français ou étrangers, des laboratoires publics ou privés. 


\title{
Excitonic mechanism of local phase transformations by optical pumping.
}

\author{
Serguei Brazovskii ${ }^{1,3,4^{*}}$ and Natasha Kirova, ${ }^{2,3}$ \\ ${ }^{1}$ LPTMS (CNRS-UMR 8626), Bat. 100, Université Paris-sud, 91405 Orsay cedex, France \\ ${ }^{2}$ LPS (CNRS-UMR 8502), Bat. 510, Université Paris-sud, 91405 Orsay cedex, France \\ ${ }^{3}$ International Institute of Physics, 59078-400 Natal, Rio Grande do Norte, Brazil \\ ${ }^{4}$ Jozef Stefan Institute, Jamova 39, SI-1000, Ljubljana, Slovenia
}

\begin{abstract}
Transformations of cooperative electronic states by impacts of optical pumping or/and electrostatic doping is a new mainstream in physics of correlated systems. Here we present a semi-phenomenological modelling of spacio-temporal effects in a system where the light absorption goes through a channel creating the excitons - intra-molecular ones or bound electron-hole pairs - and finally the condensate of optical excitons feeds and stimulates phase transformations. Interacting with a near-critical order parameter and deformations, the excitons are subject to self-trapping. That locally enhances their density which can surpass a critical value to trigger the phase transformation, even if the mean density is below the required threshold. The model can be used e.g. as a simplified version of optically induced neutralionic transitions in organic chain compounds.
\end{abstract}

Key words: optical pumping, phase transition, exciton, self-trapping,

\section{Excitons in Pump Induced Phase Transitions.}

Nowadays, commutations among cooperative electronic states can be performed by short optical pulses [1,2] which approach was baptized as "pump induced phase transitions" - PIPT [3]. Various symmetry broken ground states are being tested: crystallization [4] of electrons (charge order, also with ferroelectricity) or of e-h pairs (charge/spin density waves), the superconductivity, Peierls and Mott insulators. In many experiments, the electrons are excited to a high energy of the photon followed by a very fast and incoherent initial cooling and then by a sequence of coherent effects. Here, we consider the cases when excitons appear as main intermediate product of the light absorption which then triggers a phase transformation in another coupled degree of freedom $\eta$. That happens either when the photons are tuned in resonance with some intra-molecular excitons, or when bound e-h pairs (Wannier-Mott or chargetransfer excitons) intermittently appear after the initial cooling of hot electrons and holes. These situations were realized in experimental studies [5-7] of neutral-ionic transitions [8] which case has inspired our work.

In our scenario, the initial short pulse of photons creates a high (experimentally, up to one per a few unit cells) concentration n of excitons. Being quantum delocalized Bose particles, the excitons form a quasicondensate as a macroscopic quantum state which then evolves interacting with other degrees of freedom $\eta$ prone to a $1^{\text {st }}$ order instability. Above a certain threshold $n>n_{\mathrm{c} 1}$ the PIPT can take place homogeneously but actually it proceeds through local nucleations. Curiously, even well below the threshold $\mathrm{nc}<\mathrm{n}<\mathrm{n}_{\mathrm{c} 1}$, the transformation can take place being initiated locally; another much lower threshold $\mathrm{n}_{\mathrm{c}}<\mathrm{n}_{\mathrm{c} 1}$ needs to be

\footnotetext{
"Corresponding author, e-mail: brazov@Iptms.u-psud.fr

Pubished in: Journal of Superconductivity and Novel Magnetism 27 (2014) 1009-1013
} 
surpassed. That happens because of effect of self-trapping of the excitons [9] via their interaction with deformations $\eta$, akin to self-focusing in the nonlinear optics or to formation of polarons from electrons. The locally enhanced density of excitons $n(x)$ can surpass the critical value to trigger the phase transformation in $\eta$, even if the mean density $n_{0}$ is below the required threshold $n_{0}<n_{c 1}$. Even above the homogeneous threshold $\mathrm{n}_{\mathrm{c} 1}$, the phase transformation proceeds locally, via multiplying domains.

\section{Phenomenological approach to dynamics of photo-induced transitions.}

\section{2a. Minimal model for a multi-stable chain.}

Competition of phases in quasi-1D systems is usually discussed in terms of strings of one phase embedded to another one which is quite consistent for thermodynamic $1^{\text {st }}$ order transitions. But for PIPT, the concepts from thermodynamics and statistics are not sufficient to model the real time dynamics. Theory of optically induced transformations faces highly challenging difficulties when taken at levels of ab initio calculations or microscopic models [10,11]. Fortunately, over a long range of time scales the system behavior seems to be governed by collective variables $\eta$ like an order parameter and lattice deformations. Effectiveness of such a phenomenological approach has been proven recently, e.g. by detailed modeling of coherent dynamics of macroscopic electronic order through a symmetry breaking transition which allowed to recover such effects as dynamic symmetry breaking, stratification in domains and subsequent collapses of their walls [12,13]. Another success was a description of the recently discovered [14] switching to a truly stable hidden topologically protected quantum state of an electronic crystal (the CDW).

The first order phase transition can be described minimalistically by the free energy $\mathrm{W}(\eta)$ as a function of a single parameter $\eta$ which curve shows two minima at $\mathrm{W}_{1}=\mathrm{W}\left(\eta_{1}\right)$ and $\mathrm{W}_{2}=\mathrm{W}\left(\eta_{2}\right)$ separated by the barrier ant $\mathrm{W}_{\mathrm{b}}=\mathrm{W}\left(\eta_{\mathrm{b}}\right)$ (see Fig.1). At presence of excitons, and in a possibly inhomogeneous state, the variational energy $\mathrm{W}(\eta, \Psi)$ becomes a functional of $\eta(\mathrm{x})$ and of the excitons' common wave function $\psi(\mathrm{x})$ :

$$
\begin{gathered}
W(\eta, \Psi)=\frac{a}{2} \eta^{2}-\frac{b}{3} \eta^{3}+\frac{c}{4} \eta^{4}-\mathrm{g \eta}|\psi|^{2}+\frac{\mathrm{k}}{2}|\psi|^{4}+\frac{\hbar^{2}}{2 m}\left|\frac{\partial \psi}{\partial \mathrm{x}}\right|^{2}+\frac{a s^{2}}{2}\left(\frac{\partial \eta}{\partial \mathrm{x}}\right)^{2} \\
\int_{-L}^{L} d x|\psi|^{2}=\mathrm{N}=\mathrm{n}_{0} \mathrm{~L}
\end{gathered}
$$

Here $2 L$ is the sample size, parameter $a$ characterizes the proximity to the point of the $2^{\text {nd }}$ order phase transition $(a=0)$, $\mathrm{s}$ is the sound velocity for small perturbations of $\eta$, $\mathrm{g}$ is the constant of coupling between $\eta$ and $\psi, \mathrm{k}$ is the constant of repulsion among the excitons, $\mathrm{m}$ is the exciton mass. The parameters $b$ and $c$ will be chosen in such a way, that the energies $\mathrm{W}_{1}$ and $\mathrm{W}_{2}$ are close and interchangeable in order, and the separating barrier $\mathrm{W}_{\mathrm{b}}$ is low. With no excitons, we can choose $\eta_{1}=0, \mathrm{~W}_{1}=0$.

Enforcing the homogeneous scenario, with the self-trapping of excitons, we arrive at the following picture. (Details are given in the appendix.) Before the excitation, there are two possible states: the stable phase 1 , and the metastable one 2 separated by the barrier. At presence of excitons, both minima and the barrier decrease their values and shift their positions to higher $\eta$, e.g. $\eta_{1}>0, W_{1}<0$, see Fig.1.

There are several critical levels of the pumping density: two of a thermodynamic origin $\mathrm{n}_{\mathrm{c} 1}$ and $\mathrm{n}_{\mathrm{c} 2}$ giving criteria of the $1^{\text {st }}$ and the $2^{\text {nd }}$ order phase transitions, and one of a purely dynamic origin, $\mathrm{n}_{\mathrm{d}}$. At $\mathrm{n}_{\mathrm{c} 1}$ the energies of the two states become equal; the states are separated by the barrier. At $n_{d}$ the state 2 is well 
below in energy but the state 1 is metastatble: the barrier still exist, but its height value drops down to $\mathrm{W}_{\mathrm{b}}=0$. At $\mathrm{n}_{\mathrm{c} 2}$, the barrier disappears, the phase 1 becomes absolutely unstable; the instability position $\eta_{\mathrm{c} 2}$ $=\eta_{\mathrm{b}}$ at $\mathrm{n}_{\mathrm{c} 2}$ does not dependent on excitons' coupling parameters $\mathrm{g}$, $\mathrm{k}$.

After the excitation to a mean level $\mathrm{n}_{0}<\mathrm{n}_{\mathrm{d}}$, the energy minimum $\mathrm{W}_{1}$ drops below the initial $\mathrm{W}_{\mathrm{S}}=0$. The excess potential energy transforms into the kinetic one which initiates collective oscillations. For a sufficient dissipation, or always if $\mathrm{n}_{0}<\mathrm{n}_{\mathrm{d}}$, after a number of oscillations the system eventually can settle in the minimum corresponding to the phase 1 . But at $n_{0}>n_{d}$ and for low damping the pendulum oscillations reaches over the barrier maximum which is below the energy excess and the system can finally fall to the next minimum corresponding to the state 2 . With inevitable fluctuations of basic parameters, the system will be divided in alternating domains of coexisting phases [11,12].

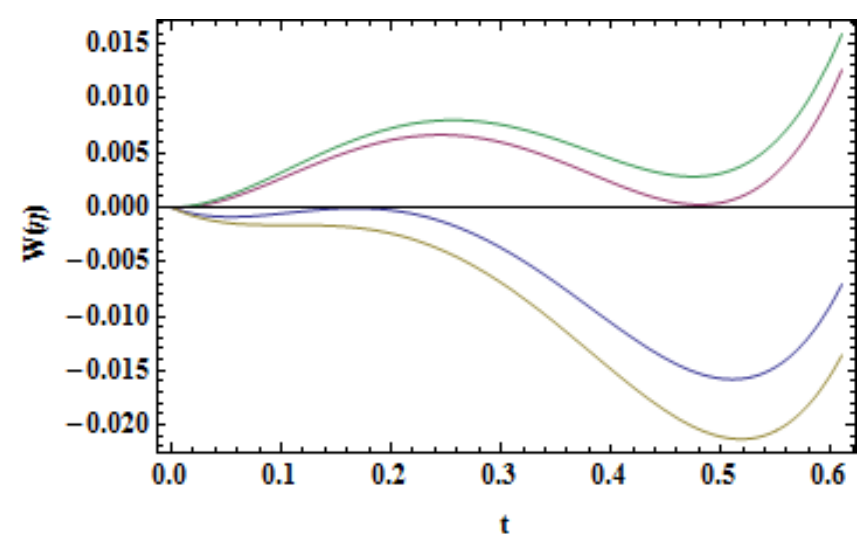

Figure 1. Evolution of the free energy shape $\mathrm{W}(\eta, \psi)$ as a function of $\eta$ at a given pumping $|\psi|^{2}=\mathrm{n}_{0}=\mathrm{cnst}$. From top to bottom: without pumping, phase 1 is stable, phase 2 is metastable; first critical pumping $\mathrm{n}=\mathrm{n}_{\mathrm{c} 1}-$ possibility for coexistence of phases 1 an 2; the dynamic threshold $\mathrm{n}=\mathrm{n}_{\mathrm{d}}$ when the barrier height drops below the energy ( $\mathrm{W}=0$ ) of the initial state; second critical pumping $n=n_{\mathrm{c} 2}-$ absolute instability of the phase 1 , only phase 2 keeps existing.

\section{2b. Modeling of self-trapping evolution.}

Now we are going to demonstrate in real time how the optical excitations provoke local transformations between two phases. We model the self-trapping process by considering the coupled space-time evolution of the exciton wave function $\psi(\mathrm{x}, \mathrm{t})$ and the order parameter $\eta(\mathrm{x}, \mathrm{t})$. We have to solve self-consistently three coupled equations: the two-component time-dependent Schrödinger equation for the wave function $\psi(\mathrm{x}, \mathrm{t})$ of the excitons together with the evolution equation for $\eta(\mathrm{x}, \mathrm{t})$.

$$
\begin{aligned}
& i \frac{\partial \psi}{\partial t}=\frac{\delta W}{\delta \psi^{*}}=-\frac{\hbar^{2}}{2 m} \frac{\partial^{2} \psi}{\partial x^{2}}-g \eta \psi+\frac{k}{2}|\psi|^{2} \psi \\
& \alpha \frac{\partial^{2} \eta}{\partial t^{2}}+\beta \frac{\partial \eta}{\partial t}=-\frac{\partial W}{\partial \eta}=\alpha s^{2} \frac{\partial^{2} \eta}{\partial x^{2}}+g|\psi|^{2}-a \eta+b \eta^{2}-c \eta^{3}
\end{aligned}
$$

Here $\alpha=a / \omega^{2}$, where $\omega$ is the bare frequency of collective oscillations near the state 1 . The system is defined on a large while finite interval $(-\mathrm{L},+\mathrm{L})$; the boundary conditions are $\psi( \pm \mathrm{L}, 0)=0, \eta( \pm \mathrm{L}, 0)=0$. Initial 
conditions for these equations are those of the equilibrium phase just after the pumping: $\eta(x, 0)=0$ - no changes yet, and $\psi(\mathrm{x}, 0)=\mathrm{n}_{0}{ }^{1 / 2} \cos (\pi \mathrm{x} / 2 \mathrm{~L})$ - the lowest wave state delocalized over the sample box (-L,L). We do not introduce any noise which will result sometimes in delayed waiting times before the selftrapping occurs; but once started, it proceeds fast corresponding to proper time scales of the equations.

Our common choice of parameters will be: $a=1, b=6, c=8.2, g=2, \mathrm{k}=1, \alpha=10^{3}, \beta=10$ unless specified.

\section{Results of numerical modeling.}

\section{3a. Selftrapping of excitons without triggering: low pumping or a mono-stable system.}

We start with a low pumping when the phase transformation does not take place yet; the system spans only the region of the local stability of the initial phase. This regime is similar to a conventional selftrapping evolution, i.e. the when $b=c=0$. The $3 \mathrm{D}$ plot for the quantum density of excitons is shown in Fig. 2 . After a waiting time $t \approx 200$, the fast self-trapping starts concentrating the wave function near the sample centre; this process is superimposed by long living breathing oscillations. These results can be applied also to formation of polarons if pumping creates electrons or holes.

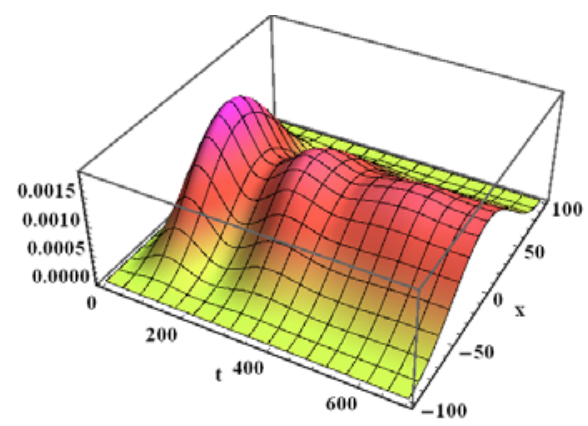

Figure 2. 3D plot of space-time evolution of the excitons' density $|\psi(\mathrm{x}, \mathrm{t})|^{2}$ for a low pumping - already a selftrapping but no switching yet.

\section{3b. Switching near the critical dynamic pumping $n_{d}$ in a bi-stable quantum dot.}

As the first example of the PIPT, we consider the switching in a quantum dot. In this case the model (5) becomes zero-dimensional - all terms with space derivatives should be omitted. The exciton wave function modulus can be only constant except for the jump to $|\psi|^{2}=\mathrm{n}_{0}=\mathrm{cnst}$ at $\mathrm{t}>0$. Then $\eta(\mathrm{t})$ is determined by the equation

$$
\alpha \frac{d^{2} \eta}{d t^{2}}+\beta \frac{d \eta}{d t}-g\left|\psi_{0}\right|^{2}+a \eta-b \eta^{2}+c \eta^{3}=0
$$

The numerical solutions of Eq. (6) are shown in Fig.3. 


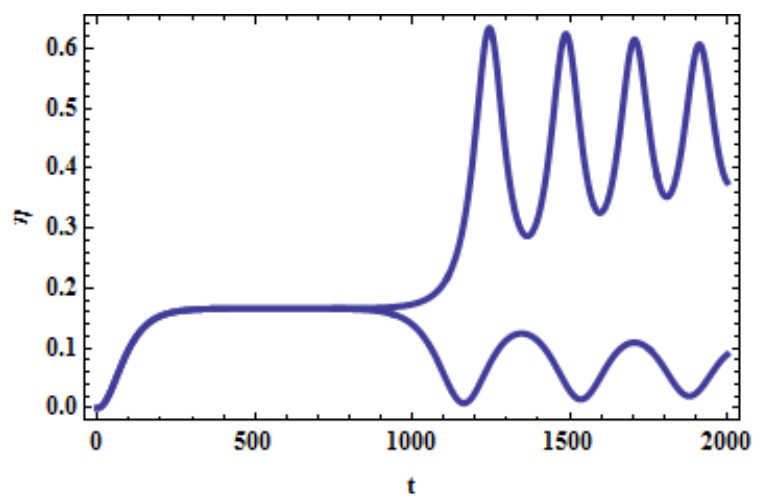

Figure 3. Zero-dimensional model of a bi-stable quantum dot. Evolutions of $\eta$ just below and above the critical dynamic pumping $\mathrm{n}_{\text {switch }}=0.187$ (Here we take $\beta=0.1$ )

Fig. 3 shows the time evolution of the order parameter just below and above the critical dynamic pumping. In both cases, the system spends a long time near the top of the energy barrier $\left(\eta_{\mathrm{b}} \approx 0.16\right)$ which results in the plateau in $\eta(t)$ at $t<1000$ on both plots in Fig. 3. At $t>1000$ for $n<n_{\text {switch }}$ the system returns to the state 1 which is displaced slightly $(\eta \approx 0.08)$ by presence of the excitons. But for $\mathrm{n}>\mathrm{n}_{\text {swith }}$ the system makes a big jump ( $\eta \approx 0.5$ ) to the phase 2 . From these calculations, we obtain a very precise determination of the critical pumping between switching and restoring regimes. Notice, that $\mathrm{n}_{\text {switch }}$ depends on the damping parameter $\beta$, being larger than the corresponding theoretical value of absolute instability $\mathrm{n}_{\mathrm{d}}=0.186$ (see Appendix).

\section{3c. Evolution and switching in an extended bi-stable system.}

Now we describe the time evolution and the switching between phases for the extended bi-stable system when the self-trapping of excitons plays the crucial role. Fig. 4 presents our main results for spaciotemporal behavior of the order parameter and the density of excitons. The three columns of the Fig.4 show results for just subcritical, critical and well supercritical values of pumping: $n=0.0058412,0.0058413$, 0.05. The switching takes place in a limited region around the sample center $\mathrm{x}=0$, and the pumping threshold $\mathrm{n}_{\text {swith }} \approx 0.006$ is more than three times lower than the dynamic critical pumping $\mathrm{n}_{\mathrm{d}} \approx 0.02$ for the homogeneous path or for a quantum dot.

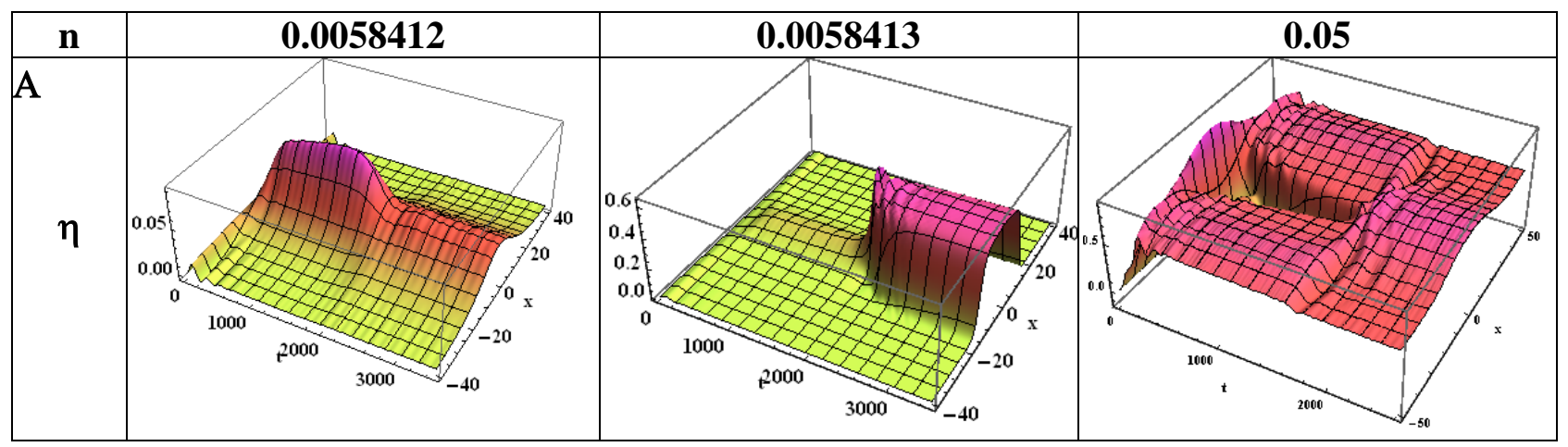




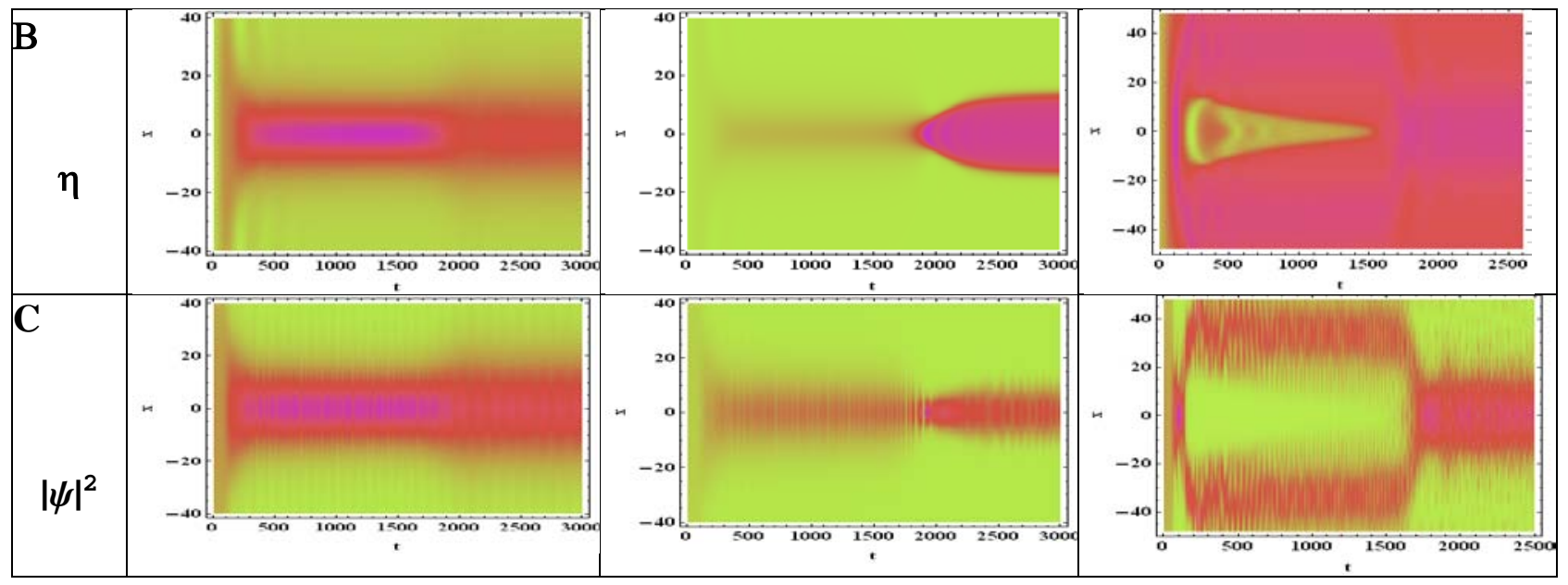

Figure 4. Modeling results for the pumping $\mathrm{n}_{0}$ just below (left), just above (center) and well above (right) the dynamic critical pumping threshold. A - 3D plot for $\eta(x, t), \mathbf{B}$ and $\mathbf{C}-2 \mathrm{D}$ density plots for $\eta(\mathrm{x}, \mathrm{t})$ and $|\psi(\mathrm{x}, \mathrm{t})|^{2}$. Notice that in 3D plot for $\eta$ the vertical scale () in the left graph is 10 times smaller than for other plots.

The self-trapping starts to develops first within the phase 1 ; after a time $\mathrm{t}=200-400$ the distributions of $|\psi(\mathrm{x})|^{2}$ and $\eta(\mathrm{x})$ are concentrated within the self-tuned localization length $\ell$ which is well smaller and almost independent of L. Becoming enhanced, $|\psi(\mathrm{x})|^{2}$ overcomes critical value which provokes the phase transition within this length. Similar to the quantum dot case, near the threshold the system spends a long time t 1500 near the barrier top, afterwards passing either back to the phase 1 or forward to the phase 2; in both cases the selftrapping is preserved. Outside the localization region, the system stays intact in the former state 1 with weakly decaying wave perturbations. Coherent oscillations with low frequencies $\omega$ of displacements and the velocity s are generated, together with strong high frequency oscillations (Rabitype) of $\psi$ due the rapid change of the exciton's potential energy by switching in the potential $\sim \eta$.

The space distribution looks simple at all times only at the threshold pumping. Below that (Fig.4, left column), $\mathrm{n}_{0}<\mathrm{n}_{\mathrm{cr}}$, the two humps with a dip in the centre develop over all the long time when the system is trying to pass over the barrier; there are only single humps before and afterwards in the phase 1 . For the high pumping $\mathrm{n}>>\mathrm{n}_{\mathrm{cr}}$ shown in the right column, the distribution also splits in two wide ones separated by a sharp dip. The split regions spread wide but after a time they merge back to the single central region. After second critical time (not shown in the Fig.4), next pairs of fringes appear in a sequence. From inclinations of their waves in the x-t plane we conclude the propagation with the sound velocity s from the equations.

\section{Conclusions}

We conclude that the excitons pumped to or relaxed at a coherent delocalized quantum state can provoke local commutations between the stable and the metastable phases and even lead to the absolute instability of the initial phase. The initial string of the new embedded phase appears as self-trapping; its length is determined by a balance between gaining the potential energy and loosing the quantum kinetic energy of the exciton. The phase transformation proceeds from large to medium distances, rather than from small to larger ones as in a rather common molecular picture of excitons. The suggested scenario looks to be a minimally sufficient one to take into account the quantum nature of the excitons and their cooperative 
effects, to understand stabilization of a long string and the efficiency of phase transformations. The results can be already applied to well studied neutral-ionic transitions. Some more elaboration of the energy functional will bring to a detailed level.

\section{Acknowledgements.}

The authors are grateful for hospitality of the Graduate School of Frontier Sciences at the University of Tokyo, where this work was initiated. We particularly acknowledge very stimulating impact from Prof. H.Okamoto and members of his laboratory, and discussions with Prof. N. Nagaosa.

\section{Appendix.}

The critical pumping $\mathrm{n}_{\mathrm{c} 1}=\left|\psi_{\mathrm{cl}}\right|^{2}$ can be found as a point where the following conditions are fulfilled:

$$
\begin{gathered}
\mathrm{W}(\eta, \psi)=\mathrm{W}(\eta, \psi) \quad \text { i.e. } \quad \frac{a}{2} \eta^{2}-\frac{b}{3} \eta^{3}+\frac{c}{4} \eta^{4}-\mathrm{g \eta}|\psi|^{2}=0, \\
\frac{\partial w}{\partial \eta}=a \eta-b \eta^{2}+c \eta^{3}-\mathrm{g}|\psi|^{2}=0, \frac{\partial^{2} W}{\partial \eta^{2}}=a-2 b \eta+3 c \eta^{2}<0
\end{gathered}
$$

The critical value $\mathrm{n}_{\mathrm{c} 2}$ for the absolute instability of the phase 1 is reached when the barrier merges the minimum corresponding the phase $1: \eta_{1}=\eta_{\mathrm{b}}=\eta_{\mathrm{c} 2}$ at $\partial \mathrm{W} / \partial \eta=0$ and $\partial^{2} \mathrm{~W} / \partial \eta^{2}=0$. We find the position and the threshold pumping as

$\eta_{c 2}=\frac{b-\sqrt{b^{2}-3 a c}}{3 c} \quad, n_{c 2}=\frac{9 a b c+2\left(b^{2}-3 a c\right)\left(\sqrt{b^{2}-3 a c}-b\right)-2 b^{3}}{27 g c^{2}}$

Choosing $a=1, b=6, c=8.2, g=2$, we obtain for the strong excitons' repulsion $(k=1): \eta_{\mathrm{d}}=0.161, \mathrm{n}_{\mathrm{d}} \approx 0.0186$, and for the weak one $(k=0.1): \eta_{\mathrm{d}}=0.163, \mathrm{n}_{\mathrm{d}}=0.0181$. For the second threshold, independently of $\mathrm{k}$ and $\mathrm{g}$ : $\eta_{\mathrm{c} 2}=0.107, \mathrm{n}_{\mathrm{c} 2}=0.0242$.

\section{References.}

[1] K. Nasu, Photoinduced phase transitions, (World Scientific, 2004).

[2] Electronic States and Phases Induced by Electric or Optical Impacts, S. Brazovskii and N. Kirova Eds., Eur. Phys. J. Special Topics, 222, Issue 5 (2013).

[3] Proceedins of the 4th International Conference Photoinduced Phase Transitions and Cooperative Phenomena, T. Luty and A. Lewanowicz Eds., Acta Physica Polonica A, 121 (2012).

[4] Proceedings of the International conference on Electronic Crystals, ECRYS-2012, S. Brazovskii, . Kirova, P. Monceau Eds., Physica B: Condensed Matter, 407 , Issue 11 (2012).

[6] H. Okamoto, "Ultrafast photoinduced phase transitions in one-dimensional organic correlated electron systems" in Molecular Electronic and Related Materials-Control and Probe with Light, 59-97, Transworld Research Network (2010).

[7] T. Miyamoto, H. Uemura, and H. Okamoto, Phys. Soc. Jpn., 81, 073703 (2012) 
[8] N. Nagaosa, Solid State Commun., 51, 179 (1986) ; J. Phys. Soc. Jpn. 55, 3488 (1986).

[9] E.I. Rashba, "Self-Trapping of Excitons” in Excitons, E.I. Rashba and M.D. Sturge Eds., (NorthHolland) 1982, p. 543; and also in Excitons, Selected Chapters, E. I. Rashba and M. D. Sturge Eds., North-Holland Personal Library (Elsevier) 1987, p.273.

[10] T. Tohyama, Eur. Phys. J. Special Topics, 222, 1065 (2013).

[11] S. Ishihara, J. Ohara and Y. Kanamori, Eur. Phys. J. Special Topics, 222 , 1125 (2013).

[12] R. Yusupov, et al, Nat. Phys. 6, 681 (2010).

[13] R. Yusupov, et al, J. Supercond. Nov. Magn., 24, 1191 (2011).

[14] L. Stojchevska, T. Mertelj, P. Kusar, I. Vaskivskyi, D. Svetin, S.Brazovskii and D. Mihailovic, under revision in Science, (2013). 